\title{
Eragrostis paniciformis (Poaceae): A new record for Asia from Kerala, India
}

\author{
J.F. Veldkamp1, Thoiba Kottekkattu² and A.K. Pradeep ${ }^{2 *}$ \\ ${ }^{1}$ Naturalis Biodiversity Center, P.O. Box 9517, 2300 RA Leiden, The Netherlands. \\ 2Department of Botany, University of Calicut, Calicut University P.O., Malappuram, Kerala - 673635, India. \\ *E-mail: akpradeep1@rediffmail.com
}

\begin{abstract}
Eragrostis paniciformis (A. Braun) Steud. (Poaceae) is reported here as a new record for Asia based on the collection made from Kerala, India. Description, illustrations and key to differentiate from its allied species, E. curvula (Schrad.) Nees are provided.
\end{abstract}

Keywords: East Africa, Eragrostis curvula, India, Kerala, Lectotypification, Poaceae

\section{Introduction}

The genus Eragrostis Wolf comprises 423 species distributed in tropical to temperate regions of the world and is found in open habitat and sometimes forests from sea level to high altitudes (Ingram \& Doyle, 2007; Chaisongkram, 2013). It is represented by 44 species in India (Vivek et al., 2015).

As part of a taxonomic revision of the subtribe Eragrostidinae in peninsular India, the authors collected an interesting specimen of an Eragrostis from the Ghat roadsides in Idukki district, Kerala. Critical studies revealed this to be an African species, E. paniciformis (A. Braun) Steud., which is a new record for Asia. It is closely similar to E. curvula but can be distinguished by its much long (up to $1 \mathrm{~m}$ ) culms, glabrous basal sheaths, caducous paleas, and terete caryopsis.

Eragrostis paniciformis (A. Braun) Steud., Syn. Pl. Glumac.: 268. 1854.

Figs. 1, 2

Poa paniciformis A. Braun, Flora 24: 274. 1841. Type: ETHIOPIA, cultivated in the Carlsruhe Botanical Garden, A. Braun s.n. (Holotype, B (lost); Lectotype (designated here): ETHIOPIA, 1841, A. Braun s.n. (K000366406, image!); Isolectotype, K000366405, image!).

Perennial. Culms tufted, erect, branching intravaginally at base, $0.15-1 \mathrm{~m}$ long, eglandular. Leaf blade $10-25 \times 0.5-1.5 \mathrm{~cm}$, flat, linear-lanceolate, slightly cordate at base, entire and eglandular at margins; acute-acuminate at apex; surface sparsely pilose above, glabrous beneath; midrib prominent. Panicles spreading or somewhat contracted, 4-9 $(-20) \mathrm{cm}$, axils of the panicle branches glabrous, branches erecto-patent, smooth or minutely scaberulous (30x), lowermost panicle branches solitary, 3-15 cm long, naked in lower $c$. 0.3th part. Pedicels $0.5-15 \mathrm{~mm}$ long, much shorter to much longer than spikelets. Spikelets laterally compressed, 2.5-14 × 2-5 mm, disarticulating from base to upwards, rachilla persistent. Glumes subequal, acute to acuminate, 1 -veined. Lower glumes $1.8-3 \mathrm{~mm}$ long, $0.75-0.9$ times as long as first lemma. Upper glumes $2-3 \mathrm{~mm}$ long. Rachilla zigzag, joints not visible between lemmas. First lemma somewhat gibbous, 2-2.7 $\mathrm{mm}$ long, apiculate, granular (30x). Paleas caducous, keels ciliolate. Anthers 3, $0.5-0.75 \mathrm{~mm}$ long, $0.25-0.3$ times as long as lemma. Caryopsis ellipsoid, 0.8$1.3 \mathrm{~mm}$ long, terete, dorsally not grooved. Pericarp smooth, tea-coloured.

Habitat: Found in seasonally wet soils in deciduous bushland, swamp grassland, stream sides, wet flushes, and roadsides between 1075 and 2300 m elevation. Associated with Chrysopogon sp., Heteropogon contortus (L.) P. Beauv. ex Roem. \& Schult., Hypericum mysurense Wight \& Arn. and Sporobolus sp.; locally common.

Specimen examined: INDIA, Kerala, Idukki district, Mattupetty, $10^{\circ} 08^{\prime} 00.0^{\prime \prime} \mathrm{N}, 077^{\circ} 12^{\prime} 35.0^{\prime \prime} \mathrm{E}, 1421 \mathrm{~m}$, 16.12.2014, Thoiba \& Pradeep CU 137599 (CALI \& $\mathrm{MH})$.

Distribution: East Africa from Sudan, Eritrea to Malawi and Zambia. Introduced in Australia (SE Queensland), Brazil, India (Kerala), Niger and Venezuela. 


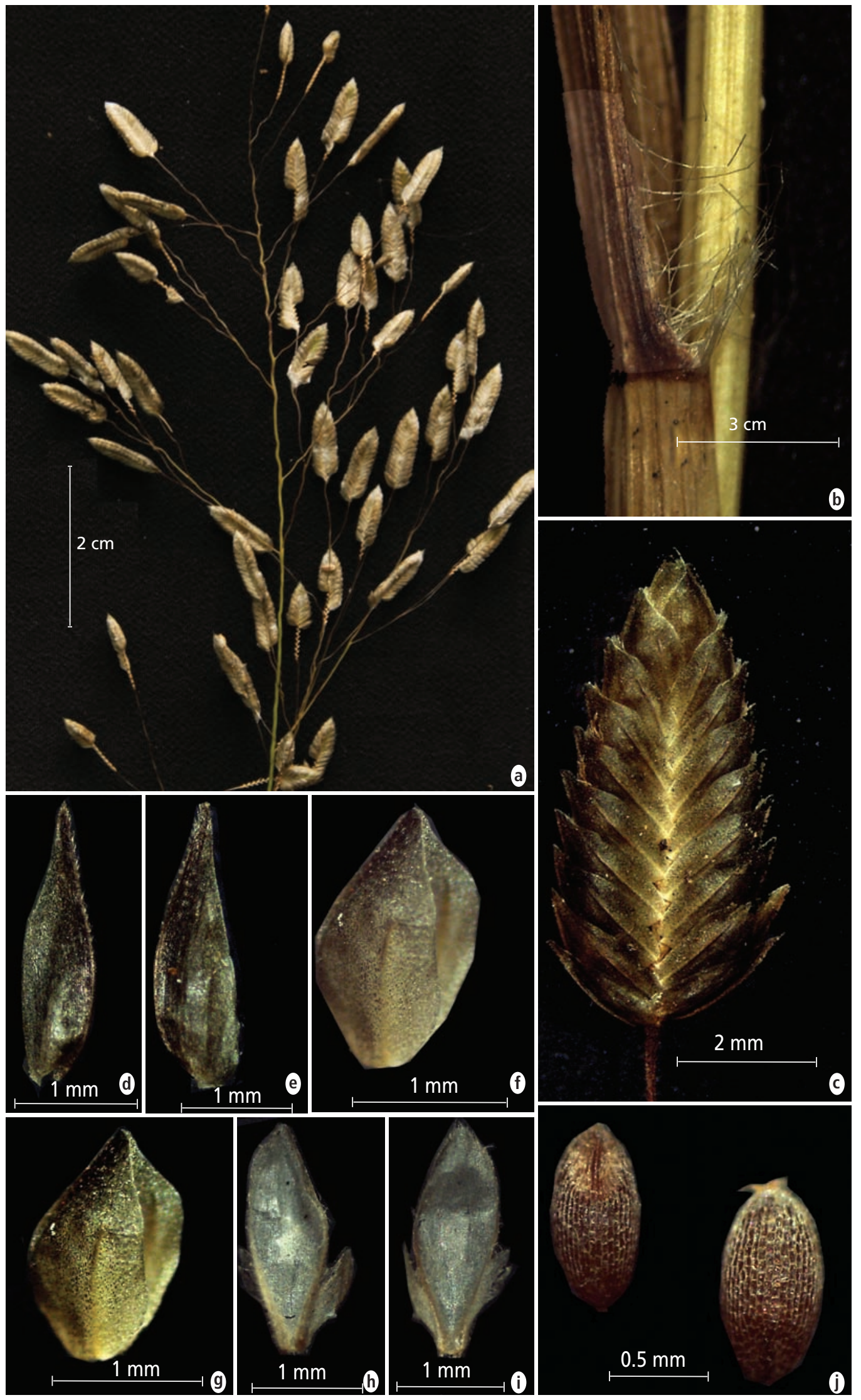

Fig. 1. Eragrostis paniciformis (A. Braun) Steud.: a. Panicle; b. Ligule; c. Spikelet; d. Lower glume; e. Upper glume; f. Floret; g. Lemma; h, i. Palea (dorsal and ventral views); j. Caryopsis. 


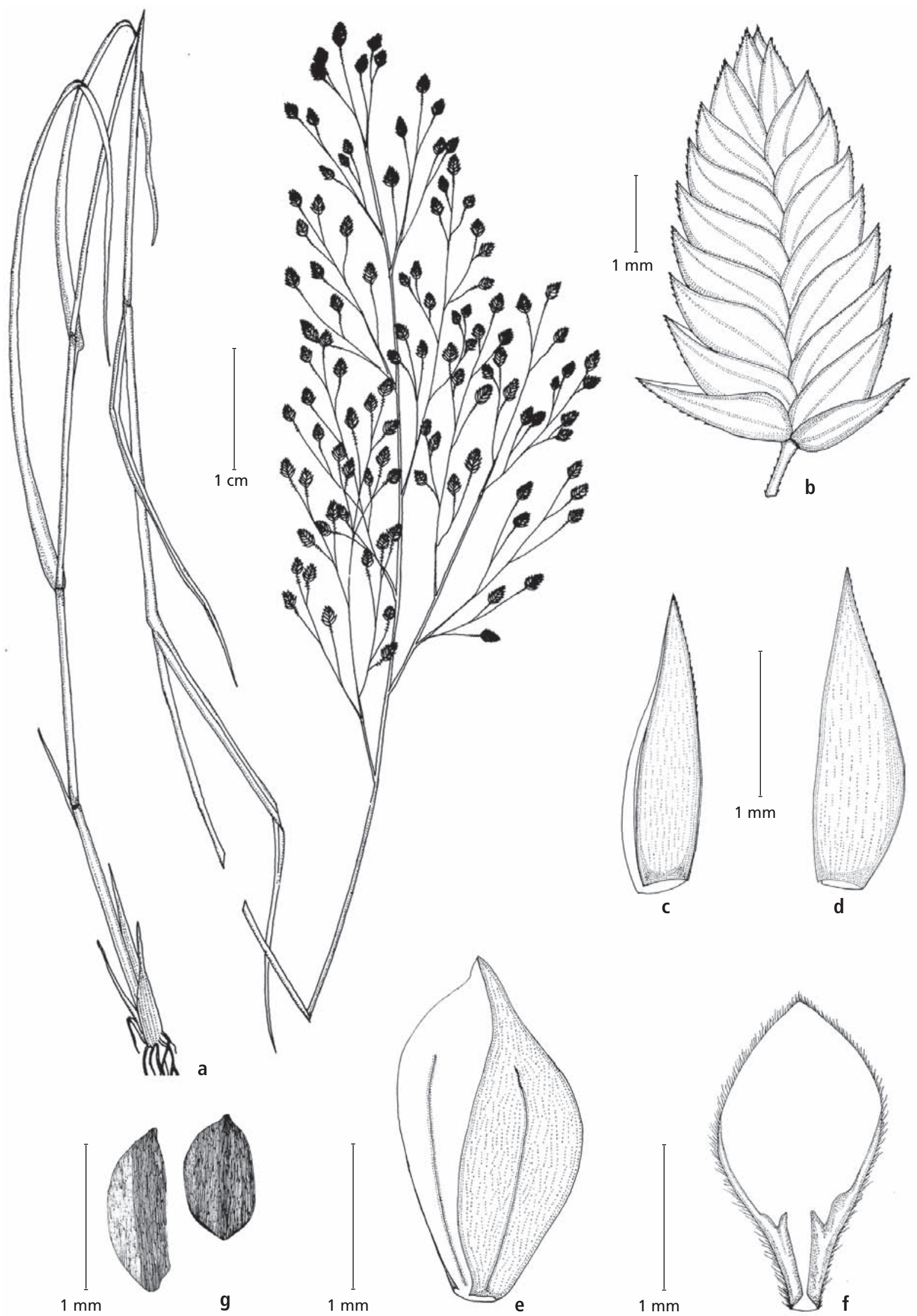

Fig. 2. Eragrostis paniciformis (A. Braun) Steud.: a. Habit; b. Spikelet; c. Lower glume; d. Upper glume; e. Lemma; f. Palea; g. Caryopsis. [Drawn by Ms. Thoiba Kottekkattu and inked by Mr. J. Van Os except 2a] 
Note: Lazarides (1997) reported that the pericarp would become loose when wetted, but we have not observed such a feature.

In Bor (1960) it keys out to E. curvula, from which it differs considerably. A key to distinguish the species is given below:

Culms 1.2-1.4 m long; basal sheaths hairy; blades $0.3-1 \mathrm{~mm}$ wide; lower axils of the panicle branches bearded at least, branches scaberulous; lower glume $1.5-1.75 \mathrm{~mm}$ long, $0.62-0.73$ times as long as first lemma; first lemma acute; paleas longpersistent, keels glabrous, smooth to scaberulous; anthers $0.9-1.25 \mathrm{~mm}$ long, $0.4-0.5$ times as long as lemma; caryopsis dorso-ventrally flattened, pericarp cinnamon-coloured

E. curvula

Culms 0.15-1 m long; basal sheaths glabrous; blades 1-15 mm wide; axils of panicle branches glabrous, branches smooth; Lower glume 1.8-3 mm long, 0.75-0.9 times as long as first lemma; first lemma apiculate; paleas caducous, keels ciliolate; anthers $0.5-0.75 \mathrm{~mm}$ long, $0.25-0.3$ times as long as lemma; caryopsis terete, pericarp teacoloured

E. paniciformis

\section{Acknowledgements}

The help rendered by the Kerala Forest Department, Govt. of Kerala, for the collection of specimens is gratefully acknowledged.

\section{Literature Cited}

Bor, N.L. 1960. The Grasses of Burma, Ceylon, India, and Pakistan. Pergamon Press, New York.

Chaisongkram, W., Chantaranothai, P. \& T.R. Hodkinson 2013. A taxonomic revision of the genus Eragrostis in Thailand. Sci. Asia 39: 111-123.

Ingram, A.L. \& J.J. Doyle 2007. Eragrostis (Poaceae): Monophyly and infrageneric classification. Aliso 23: 595-604.

Lazarides, M. 1997. A revision of Eragrostis (Eragrostideae, Eleusininae, Poaceae) in Australia. Austral. Syst. Bot. 10: 89, 138-139.

Vivek, C.P., Murthy, G.V.S., Gnanasekaran, G., Kabeer, K.A.A. \& V.J. Nair 2015. A study on the caryopses morphology of the grass genus Eragrostis in India. Nelumbo 57: 1-10.
Received: 27.11.2015

Revised and Accepted: 5.4.2017 